\title{
WAVE PATTERN OF FLOWING SNOW SLABS
}

\author{
$B y$ T. E. LANG \\ (Department of Civil Engineering and Engineering Mechanics, College of Engineering, \\ Montana State University, Bozeman, Montana 597 I5, U.S.A.)
}

\begin{abstract}
Equations are derived, using a total potential-energy formulation, which predict the wavelength of the dominant wave pattern of a flowing snow slab. Account is taken of the visco-elastic properties of an assumed surface layer of snow which overlays a distinct visco-elastic foundation layer of snow. The predicted wave pattern is recognized as a precursory mechanism which influences the characteristics of the sawtooth flank fracture pattern that may remain following a snow avalanche, particularly on broad, uniform slopes. The parameters that influence the wave pattern are determined by an order-of-magnitu de reduction of the terms in the governing differential equation for visco-elastic slab response.

RÉsumé. Comprtement ondulatoire des plaques de neige en mouvement. En utilisant une expression de l'énergie totale potentielle on établit des équations qui prévoient la longueur d'onde de l'ondulation dominante d'une plaque de neige en cours d'écoulement. On prend en compte les propriétés visco-élastiques d'un éventuel niveau superficiel de neige qui recouvrirait une couche neigeuse de base, visco-élastique distincte. Le comportement ondulatoire prévu est reconnu comme un mécanisme précurseur qui influe sur les caractéristiques de la cassure latérale en dents de scie qui peut rester après une avalanche, en particulier sur les larges versants, uniforme. Les paramètres qui influent sur le comportement ondulatoire sont déterminés par la réduction d'un ordre de grandeur des termes de l'équation différentielle qui régit la réponse visco-élastique des plaques.

Zusammenfassung. Wellenstruktur von fliessenden Schneetafeln. Mit Hilfe einer vollständigen Erfassung der potentiellen Energie werden Gleichungen zur Vorhersage der Wellenlänge der vorherrschenden Struktur in einer fliessenden Schneetafel hergeleitet. Dabei werden die viskoelastischen Eigenschaften einer angenommenen Deckschicht von Schnee, die eine im wesentlichen viskoelastische Grundschicht von Schnee überlagert, berücksichtigt. Die vorher berechnete Wellenstruktur lässt sich als Vorläufer-Mechanismus beschreiben, der die Charakteristiken des Sägezahn-Bruchmusters an den Flanken beeinflusst, das nach Abgang einer Schneelawine, besonders an breiten, gleichförmigen Hängen, zurückbleiben kann. Die Parameter, die die Wellenstruktur beeinflussen, werden aus der Reduktion der Ausdrücke in der entscheidenden Differentialgleichung für die viskoelastische Reaktion der Tafel um eine Grössenordnung gewonnen.
\end{abstract}

\section{INTRODUCTION}

A sloping snow slab, driven by gravitational force, is known to have visco-elastic material properties that admit to development of a wave pattern in time (Lang and others, I973). It has also been determined that the wave formation process takes periods of time on the order of days or weeks. The process can only occur with weak layer stratification in the slab. Maximum amplitudes of the waves are not known, but are assumed small, and are thought to be significant in establishing the characteristics of the fracture escarpment following avalanche release. The particular escarpment geometry of interest is the sawtooth pattern seen on the flanks of broad post-avalanche slopes. Many of the sawtooth patterns appear uniform, which suggests that their origin may be related to a natural wave pattern of the slab that develops as a result of flow. The intention of the present work is to set up an analytical model of a snow slab and evaluate the range and functional dependence of the predicted dominant wavelength of the slab wave pattern. In this formulation a surface layer of the slab is represented by a visco-elastic model which includes the influence of distortional as well as dilatational deformation modes. A subsurface or foundation snow layer is also represented by a visco-elastic model, which may have different material constants from those of the surface layer. It is admitted that the foundation layer may itself be a depth-hoar layer, a weak interstitial layer, or a layer of regular mid-pack snow. The analysis, lacking, as it does, specific slope parameters, is based upon the use of nominal snow visco-elastic coefficients and typical alpine slope geometries. This order-of-magnitude analysis is intended to establish the range of the pertinent parameters, which can be generally compared to the observed phenomenon in the field. 


\section{MATHEMATIGAL REPRESENTATION OF SLOPE}

A potential-energy approach is used in the calculation of the dominant wave pattern. Thus, it is necessary to specify a modal function to represent the shape of the wave pattern. In formulating a suitable function we refer to the photograph of a slope shown in Figure $\mathbf{I}$. Lines of failure show in the photograph; these are a direct result of slab deformation due to explosive control (in the foreground). The nominal periodicity, curvature, and region of greatest slope change are represented approximately by the displacement function

$$
v(x, y)=r\left[\mathrm{I}-\cos \left(\frac{2 \pi x}{l}-z\right)\right]
$$

where

$$
\left.\begin{array}{l}
r=A\left[\mathrm{I}+\cos \frac{\pi y}{b}\right], \\
z=\lambda\left[\frac{y}{b}-\frac{2}{\pi} \sin \frac{\pi y}{2 b}\right] .
\end{array}\right\}
$$

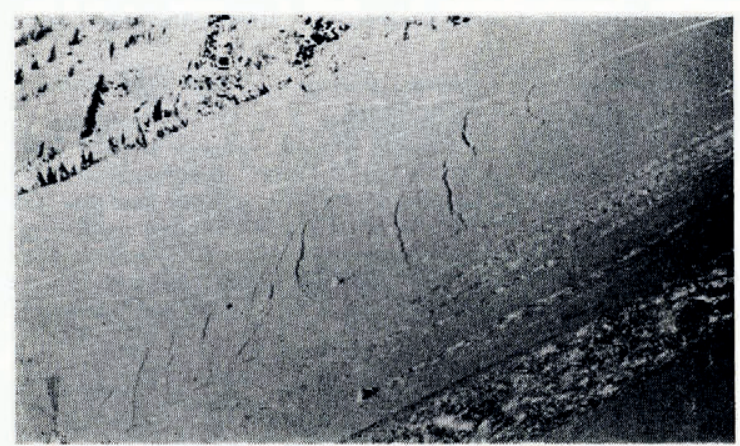

Fig. I. Post-control slope showing lines of local failure from slab deformation (Alta, Utah). (Photograph courtesy of R. I. Perla.)

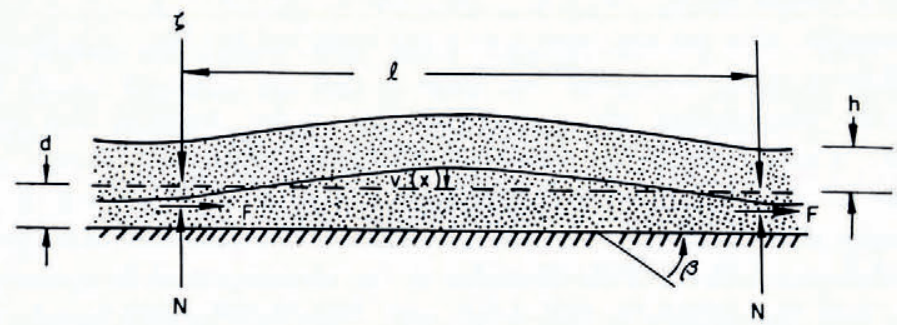

Fig. 2. A typical slope cross-section. $\left(\mathcal{N}=\frac{\gamma h l}{2} \cos \beta ; F=\frac{\eta \gamma h l}{2} \sin \beta ; \eta=\right.$ shear transfer function.)

In Equation (I) $A$ is the amplitude coefficient; $b$ is the semi-width of the deforming region of the slope; $l$ is the wavelength of the wave pattern (Fig. 2); and $\lambda$ is a phase parameter relating to the difference in displacement at $y=0$ and $y=b$. Plotting Equation (I) for the case $\lambda=\pi$, we obtain the shape shown in Figure 3 , where the in-plane slope $\theta$ is maximum at $y=b$ and zero at $y=0$. It is noted, for $\lambda=\pi$, that the displacement of the center of the slab relative to the flanks is 0.18 of the wavelength $l$. Having established a plausible displacement function, we consider next the energy and work terms of the total potential energy. 


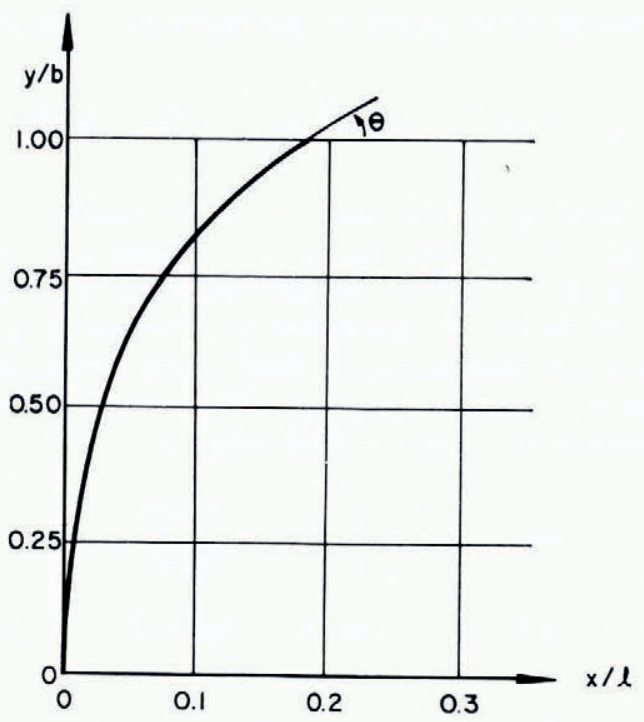

Fig. 3. The cross-slope shape of the assumed displacement function. $\left(\lambda=\pi ; \tan \theta=\frac{2 \pi b}{\lambda l}\right.$. $)$

The strain energy is assumed to be due to bending of the slab, then

$$
U=\frac{D}{2} \int_{-b}^{b} \int_{0}^{l}\left\{\left(v_{x x}+v_{y y}\right)^{2}-2(\mathrm{I}-v)\left[v_{x x} v_{y y}-v_{x y} v_{x y}\right]\right\} \mathrm{d} x \mathrm{~d} y,
$$

where the subscripts in this equation denote partial derivatives. Substituting Equation (I) into (2) and performing the indicated integrations leads to

$$
U=\frac{\pi^{4} D A^{2}}{2 h^{2} \delta^{3} \Omega^{3}}\left[24 \Omega^{4}+4 \Omega^{2} \delta^{2}+0.272 \zeta^{2} \Omega^{2} \delta^{4}+\mathrm{I} \cdot 5^{\delta^{4}}+0.13 \mathrm{I} \zeta^{2} \delta^{4}+0.0048 \zeta^{4} \delta^{4}\right],
$$

where

$$
\begin{aligned}
& \delta=\frac{l}{h}=\text { ratio of wavelength to depth of surface slab (primary unknown), } \\
& \Omega=\frac{b}{h}=\text { ratio of semi-width to depth of surface slab, } \\
& \zeta=\frac{\lambda}{\pi}=\text { normalization of displacement phase parameter. }
\end{aligned}
$$

Work by the edge friction force $F$ (Fig. 2), assuming that the shear stress fraction $\eta$ is transferred to the foundation slab, is

$$
W_{\mathrm{I}}=-\frac{\eta}{2} \int_{-b}^{b} \int_{0}^{l} F\left(\frac{\mathrm{d} v}{\mathrm{~d} x}\right)^{2} \mathrm{~d} x \mathrm{~d} y=-\frac{3}{2} \gamma h \pi^{2} b \eta A^{2} \sin \beta .
$$

Work done by the transverse component of the gravitational body force is

$$
W_{2}=-\gamma h \cos \beta \int_{-b}^{b} \int_{0}^{l} v \mathrm{~d} x \mathrm{~d} y=-2 \gamma h l b A \cos \beta .
$$


Work done by the component of the gravitational body force parallel to the slope is

$$
W_{3}=\frac{1}{2} \gamma h \sin \beta \int_{-b}^{b} \int_{0}^{l}(l-x)\left(\frac{\mathrm{d} v}{\mathrm{~d} x}\right)^{2} \mathrm{~d} x \mathrm{~d} y=\frac{3}{2} \gamma h b \pi^{2} A^{2} \sin \beta .
$$

We next assume no build-up in the force resultant parallel to the slope with distance down-slope. The rationale for this assumption stems from an observation of conditions in Figure I where, because of the separation between sections, each section must be assumed to be self-supporting. This should be the case before any fracture as well as afterwards. For the equilibrium of each section, a tension along the flank edges that balances the incomplete shear transfer to the foundation layer must be assumed. The work term, assuming that the tension resultant is uniformly distributed along the flank edge, is

$$
W_{4}=-\frac{\mathrm{I}}{2} \int_{-b}^{b} \int_{0}^{l} T \sin \theta\left(\frac{\mathrm{d} v}{\mathrm{~d} y}\right)^{2} \mathrm{~d} x \mathrm{~d} y,
$$

where $T \sin \theta=(\mathrm{I}-\eta) \gamma h b \sin \beta \cdot \tan \theta$ and $\theta$ is defined in Figure 3. We obtain, after integration,

$$
W_{4}=-\frac{3}{2}(\mathrm{I}-\eta) \gamma h l \pi^{2} \sin \beta\left(\frac{\Omega}{\zeta \delta}\right) A^{2}\left(\mathrm{I}+0.023 \zeta^{2}\right) .
$$

The physical condition that each wavelength is independently supported implies that stresses in the plane do not increase with distance down-slope, so that the strain energy of bending dominates the strain energy due to loading in the plane.

Finally, we introduce a strain-energy term corresponding to an elastic foundation. If the material modulus is specified by $E^{\star}$ then the strain energy is

$$
U_{\mathrm{f}}=\frac{E_{\mathrm{f}}}{2 d^{2}} \iiint_{V} v^{2} \mathrm{~d} x \mathrm{~d} y \mathrm{~d} z=A^{2} \frac{E_{\mathrm{f}}}{2 d}\left(\frac{9 b l}{2}\right),
$$

where $E_{\mathrm{f}}=\frac{E^{\star}(\mathrm{I}-v)}{(\mathrm{I}+v)(\mathrm{I}-2 v)}$, based upon a plane strain condition in the $x$ and $y$ directions, and $d$ is the depth of the foundation layer.

The total potential energy is then

$$
\Pi=U+U_{\mathrm{f}}-W_{\mathrm{I}}-W_{2}-W_{3}-W_{4},
$$

which, in expanded form, is

$$
\Pi=\frac{E \pi^{4} A^{2}}{24\left(\mathrm{I}-\nu^{2}\right)} R_{\mathrm{I}}+\frac{9}{4} E_{\mathrm{f}} A^{2} \delta^{4} \psi \Omega^{4}-\frac{\mathrm{I}}{2} A^{2} R_{2}+2 \gamma h A \delta^{3} \Omega^{4} l \cos \beta,
$$

where

$$
\begin{aligned}
R_{\mathrm{I}} & =24 \Omega^{4}+4 \Omega^{2} \delta^{2}+0.272 \zeta^{2} \Omega^{2} \delta^{2}+\mathrm{I} .5^{\delta^{4}}+0 . \mathrm{I} 3 \mathrm{I} \zeta^{2} \delta^{4}+0.0048 \delta^{4} \zeta^{4}, \\
R_{2} & =3 \gamma h \delta^{3} \Omega^{4} \pi^{2} A^{2} \sin \beta\left[\mathrm{I}-\frac{\mathrm{I}}{\zeta}\left(\mathrm{I}+0.023 \zeta^{2}\right)\right], \\
\psi & =h / d .
\end{aligned}
$$

We next form $\frac{\partial \Pi}{\partial A}=0$ which yields an elasticity equation for $A$ :

$$
\frac{E \pi^{4}}{\mathrm{I} 2\left(\mathrm{I}-\nu^{2}\right)} A R_{1}+\frac{9}{2} E_{\mathrm{f}} \delta^{4} \psi \Omega^{4} A-R_{2} A+2 \gamma h \delta^{3} \Omega^{4} l \cos \beta=0 .
$$


In Equation (9), $A \rightarrow \infty$ is a classical buckling condition which occurs for values of $\delta>20$, representing an extremely long wavelength. This type of wave is a possible slope response mode, but not the dominant modal condition. To determine dominant modal response we convert Equation (9) to a visco-elastic model of the slab.

\section{Dominant modal Response of a VISco-elastic SLAB}

The material of the slope is recognized to be largely in compression due to the gravitational loading. Since both dilatational and distortional mechanisms are significant with snow, neither will be neglected. However, in the absence of specific material definition, we approximate the two mechanisms by making them have equal effect. One physical result of this is that the visco-elastic equivalent of Poisson's ratio goes to zero, a near physical condition at low creep rates of snow (Brown and Lang, 1973). Using a four-element Maxwell-Voigt model, the differential operators of the constitutive law are

$$
\left.\begin{array}{l}
P=p_{2} \frac{\mathrm{d}^{2}}{\mathrm{~d} t^{2}}+p_{1} \frac{\mathrm{d}}{\mathrm{d} t}+\mathrm{I}, \\
Q=q_{2} \frac{\mathrm{d}^{2}}{\mathrm{~d} t^{2}}+q_{\mathrm{I}} \frac{\mathrm{d}}{\mathrm{d} t},
\end{array}\right\}
$$

with

$$
\begin{array}{ll}
p_{\mathrm{I}}=\frac{C_{1}}{K_{\mathrm{I}}}+\frac{C_{2}}{K_{2}}+\frac{C_{\mathrm{I}}}{K_{2}}, & q_{\mathrm{I}}=C_{\mathrm{I}}, \\
p_{2}=\frac{C_{\mathrm{I}} C_{2}}{K_{\mathrm{I}} K_{2}}, & q_{2}=\frac{C_{1} C_{2}}{K_{2}} .
\end{array}
$$

$C_{1}$ and $K_{1}$ are the Maxwell elements, and $C_{2}$ and $K_{2}$ are the Voigt elements.

The operators of Equation ( IO) are used for both the distortional (') and dilatational (") mechanisms of deformation. In the general case the correspondences as derived by Flügge ( 1967 ) become

$$
\begin{gathered}
E \rightarrow \frac{3 Q^{\prime} Q^{\prime \prime}}{{ }_{2}^{\prime} P^{\prime} Q^{\prime \prime}+Q^{\prime} P^{\prime \prime}}, \\
\nu \rightarrow \frac{P^{\prime} Q^{\prime \prime}-Q^{\prime} P^{\prime \prime}}{{ }_{2} P^{\prime} Q^{\prime \prime}+Q^{\prime} P^{\prime \prime}} .
\end{gathered}
$$

In the present case of equal participation of the two mechanisms

$$
E \rightarrow \frac{Q}{P}, \quad \nu \rightarrow \text { o. }
$$

Finally, we establish a separate set of operators for the foundation slab in which the physical coefficients are subscripted f. Substituting the operator correspondences into Equation (9) and performing the indicated differentiations, results in the following ordinary differential equation:

$$
S_{\mathrm{I}} \frac{\mathrm{d}^{4} A}{\mathrm{~d} t^{4}}+S_{2} \frac{\mathrm{d}^{3} A}{\mathrm{~d} t^{3}}+S_{3} \frac{\mathrm{d}^{2} A}{\mathrm{~d} t^{2}}+S_{4} \frac{\mathrm{d} A}{\mathrm{~d} t}+S_{5} A=-2 \gamma h \delta^{3} \Omega^{4} l \cos \beta
$$

where 


$$
\begin{aligned}
& S_{\mathrm{I}}=\frac{\pi^{4} R_{1}}{\mathrm{I} 2} p_{\mathrm{f}_{2}} q_{2}+\frac{9}{2} \delta^{4} \psi \Omega^{4} p_{2} q_{\mathrm{f}_{2}}-R_{2} p_{\mathrm{P}_{2}} p_{2}, \\
& S_{2}=\frac{\pi^{4} R_{1}}{12}\left(p_{\mathrm{f}_{2}} q_{\mathrm{I}}+p_{\mathrm{f}_{1}} q_{2}\right)+\frac{9}{2} \delta^{4} \psi \Omega^{4}\left(p_{2} q_{\mathrm{f}_{1}}+p_{1} q_{\mathrm{f}_{2}}\right)-R_{2}\left(p_{\mathrm{f}_{2}} p_{\mathrm{I}}-p_{\mathrm{f}_{1}} p_{2}\right), \\
& S_{3}=\frac{\pi^{4} R_{1}}{\mathrm{I} 2} q_{2}+\frac{9}{2} \delta^{4} \psi \Omega^{4}\left(p_{\mathrm{I}} q_{\mathrm{f}_{1}}+q_{\mathrm{f}_{2}}\right)-R_{2}\left(p_{\mathrm{f}_{2}}+p_{\mathrm{f}_{1}} p_{\mathrm{I}}+p_{2}\right), \\
& S_{4}=\frac{\pi^{4} R_{1}}{12} q_{1}+\frac{9}{2} \delta^{4} \psi \Omega^{4} q_{\mathrm{f}_{1}}-R_{2}\left(p_{\mathrm{f}_{1}}+p_{\mathrm{I}}\right), \\
& S_{5}=-R_{2} .
\end{aligned}
$$

A solution to the homogeneous part of Equation (I2) is assumed of the form $A=\mathrm{e}^{m t}$ from which the characteristic polynomial equation is obtained as

$$
m^{4}+\frac{S_{2}}{S_{\mathrm{I}}} m^{3}+\frac{S_{3}}{S_{\mathrm{I}}} m^{2}+\frac{S_{4}}{S_{\mathrm{I}}} m+\frac{S_{5}}{S_{\mathrm{I}}}=0 .
$$

To proceed further we must establish nominal values for the physical parameters. The follow. ing values are assumed:

$$
\begin{aligned}
& \text { nominal weight of a snow column in the surface slab: } \quad \gamma h=3000 \mathrm{~N} \mathrm{~m}^{-2} \\
& \text { slope angle: } \quad \beta=40^{\circ} \\
& \text { ratio of slab semi-width to surface layer thickness: } \quad \Omega=b / h=10 \\
& \text { shear transfer fraction: } \quad \eta=0.5 \\
& \text { ratio of surface-to-foundation thickness: } \\
& \text { slab center-to-flank displacement phase parameter: } \\
& \psi=h / d=\mathbf{I} . \mathbf{O} \\
& \zeta=\lambda / \pi=\mathbf{r} \text {.o. }
\end{aligned}
$$

Nominal values of the visco-elastic model coefficients are taken from Shinojima (1967) as:

$$
\begin{array}{ll}
K_{1}=30 \times 10^{5} \mathrm{~N} \mathrm{~m}^{-2}, & C_{1}=25 \times 10^{5} \mathrm{~N} \mathrm{~h} \mathrm{~m}^{-2}, \\
K_{2}=50 \times 10^{5} \mathrm{~N} \mathrm{~m}^{-2}, & C_{2}=40 \times 10^{5} \mathrm{~N} \mathrm{~h} \mathrm{~m}^{-2} .
\end{array}
$$

The orders-of-magnitude of the coefficients $S_{i}$ of the polynomial equation are evaluated, using these selected numerical values, and then substituted into Equation (14) with the following order established:

$$
m^{4}+O(\mathrm{I}) \cdot m^{3}+O(\mathrm{I}) \cdot m^{2}+O(\mathrm{I}) \cdot m-O\left(\mathrm{IO}^{-3}\right)=0 .
$$

We want the positive real roots of Equation (15) for which there is only one. This root is defined by the two lowest-order terms in the equation. The real positive root $m$ is, within an accuracy of $\mathrm{IO}^{-3}$ : I (Equation (14))

$$
m=-\frac{S_{5}}{S_{4}}
$$

Dominant modal response is determined by forming $\frac{\partial m}{\partial \delta}=0$ and solving for $\delta$. The governing equation from which $\delta$ is evaluated is, using the expressions for $S_{4}$ and $S_{5}$, which contain $\delta$,

$$
\begin{array}{r}
24 \Omega^{4}+\mathrm{I} .33 \Omega^{2} \delta^{2}+0.09 \mathrm{I} \zeta^{2} \Omega^{2} \delta^{2}-0.5 \delta^{4}-0.043 \zeta^{2} \delta^{4}-0.0016 \zeta^{4} \delta^{4}- \\
-\frac{18}{\pi^{4}} \delta^{4} \psi \Omega^{4} \frac{q_{\mathrm{P}_{1}}}{q_{\mathrm{I}}}=0 .
\end{array}
$$


Further order-of-magnitude consideration of this Equation reveals that the first and last terms are significantly greater $\left(O\left(\mathrm{IO}^{2}\right)\right)$ than any of the other terms, so that a sufficiently accurate solution for $\delta$ is simply

$$
\delta=\frac{l}{h}=3.3^{8}\left(\frac{q_{\mathrm{I}}}{\psi q_{\mathrm{f}_{\mathrm{I}}}}\right)^{\frac{1}{4}}=3.3^{8}\left(\frac{d q_{\mathrm{I}}}{h q_{\mathrm{f}_{\mathrm{I}}}}\right)^{\frac{1}{4}} .
$$

Thus, the wavelength is proportional to the surface slab depth $h$ and to the fourth root of the ratio of surface to foundation layer thicknesses (the ratio of surface layer to foundation layer Maxwell viscosities divided by $\psi$ ). It is noted that large changes in the $K_{i}$ and $C_{i}$ have negligible effect on changing the form of Equation (18), since these coefficients appear as ratios in the earlier equations.

\section{Summary AND GONCLUSIONS}

Several physically meaningful cases can be interpreted using Equation (17). The first is when thicknesses $d$ and $h$ are of the same order, but $q_{\mathrm{P}_{\mathrm{I}}} \ll q_{\mathrm{I}}$, as may be the case with a weak basal layer. For this case $l$ may be an order-of-magnitude or more larger than $h$. A second possibility is $d \ll h$, as in the case of an interstitial weak layer, but then also $q_{\mathrm{f}_{\mathrm{I}}} \ll q_{\mathrm{I}}$ is likely, and $l$ remains a multiple of $h$. Yet a third possibility is a surface slab in which $d \gg h$, but then $q_{\mathrm{I}} \ll q_{\mathrm{P}_{\mathrm{I}}}$ is likely, and $l$ should remain a multiple of $h$. Thus it is concluded from the characteristics of Equation ( 18 ) that the wavelength of a sawtooth pattern is most likely to be a multiple of the surface slab thickness depending upon the relative viscosities and thicknesses of the participating layers.

It is noted that $\Omega$, the semi-width to depth ratio, does not appear in Equation ( 18 ). This is attributed to the fact that each unit width of slope acts essentially independently of its neighboring unit widths, since the dominant terms retained from Equation ( 17 ) are the strain energies of bending along the slope and of deformation of the foundation layer. The condition that only strain-energy terms come into the specification of $\delta$ is a property of the visco-elastic response in that only the viscous flow terms influence the wavelength, and not the gravity loading. This is in contrast to the classical buckling calculation which is derived from Equation (9), in which the gravitational loading influences the stability condition. In fact, the result of Equation (18) should be viewed physically as a condition by which a slope tends to flow into a wave pattern as a result of the viscous properties of the material. However, the associated interpretation that the phenomenon is a stability condition does not follow. It is only in an infinite time (mathematically speaking) that infinite amplitudes develop under conditions associated with the derivation of Equation (18).

The major deduction from this analysis is the physical condition that the visco-elastic flow of a snow slab supported on a visco-elastic foundation has a natural tendency to form a

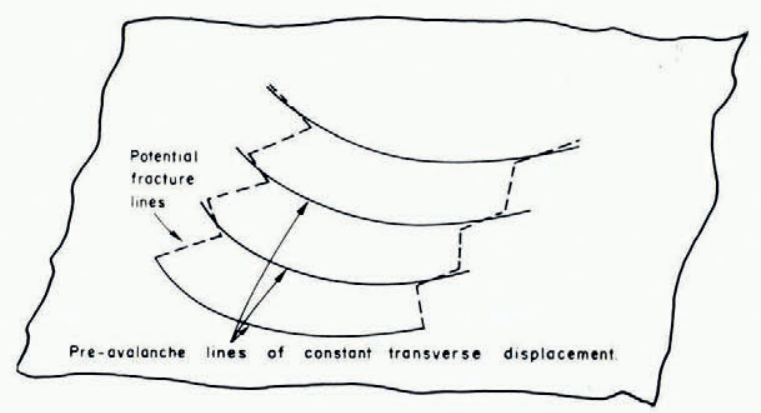

Fig. 4. The pre-avalanche wave patterns of the flowing snow slab, and hypothetical fracture lines. 
wave pattern of wavelength dependent upon specific material properties. This wave pattern, established from flow, must then influence strongly the fracture pattern that develops when the slab avalanches (Fig. 4) as is the case in numerous examples of structural failure mechanisms. For broad, uniform slopes, a regular sawtooth pattern along the flank edges of an avalanche run-out zone may be in evidence (Fig. 5). For narrow shoot-type run-out zones, local intrusions of rock outcrops or trees may obliterate or distort the sawtooth patterns (Fig. 6). The analysis carried out here can only be applied to the case of uniform slope, as no provision is made for the effects of local intrusions or non-uniform weak-layer distributions.

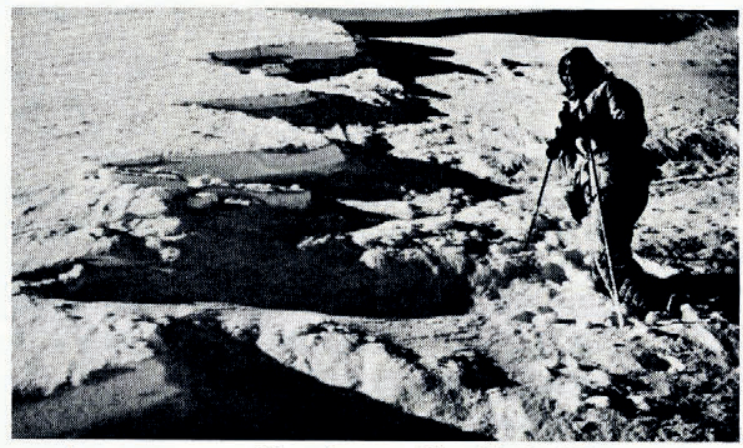

Fig. 5. A regular sawtooth pattern on a uniform slope at Alta, Utah. (Photograph courtesy of R. I. Perla.)

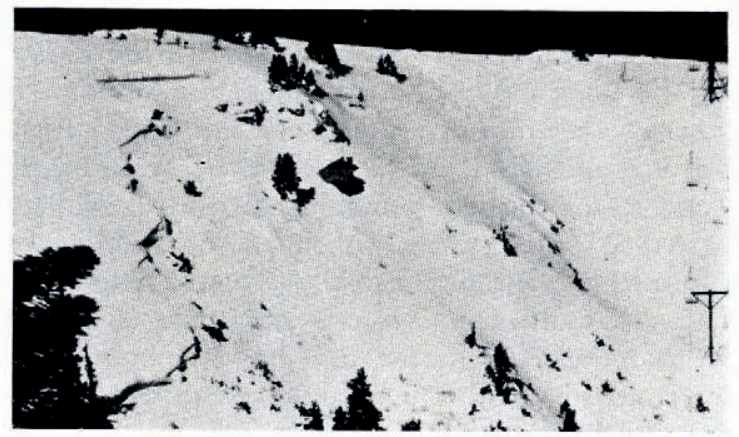

Fig. 6. An irregular sawtooth pattern controlled by local intrusions at Berthoud Pass, Colorado. (Photograph courtesy of R. A. Sommerfeld.)

In any application of Equation (18) a measurement of nominal surface and foundation layer viscosity coefficients and thicknesses is unlikely, since the boundaries where the sawtooth patterns are evident undoubtedly have different material properties from those in the preavalanche zone for the edges to have occurred at these stations. However, a reverse application of Equation ( 18) may be warranted under certain field conditions. It may be possible to measure a nominal sawtooth wavelength, estimate the surface layer and foundation layer thicknesses, and then use Equation (I8) to compute the viscosity ratio $q_{\mathrm{I}} / q_{\mathrm{p}_{\mathrm{r}}}$. Knowledge of the value of this ratio establishes the degree of weakness of the interstitial or foundation layer upon which an avalanche flows, compared to the average viscosity of the surface layer. Before one can decide whether this is or is not plausible a technique, field data on sawtooth and layering properties must be collected for a number of naturally occurring cases, so that trends in the data can be established. 


\section{REFERENCES}

Brown, R. L., and Lang, T. E. 1973. On the mechanical properties of snow and its relation to the snow avalanche. Proceedings of the eleventh Annual Engineering Geology and Soils Engineering Symposium, 1973, p. 19-36.

Flügge, W. 1967 . Viscoelasticity. Waltham, Mass., Blaisdell.

Lang, T. E., and others. 1973. Buckling characteristics of a sloping snow slab, by T. E. Lang and R. L. Brown, W. F. St. Lawrence and C. C. Bradley. Journal of Geophysical Research, Vol. 78, No. 2, p. 339-5I.

Shinojima, K. 1967. Study on the visco-elastic deformation of deposited snow. (In Oura, H., ed. Physics of snow and ice: international conference on low temperature science. . . I966. . . Proceedings, Vol. 1, Pt. 2. [Sapporo], Institute of Low Temperature Science, Hokkaido University, p. 875-907.)

\section{DISGUSSION}

D. M. McGlung: According to the ideas of progressive failure of snow slabs as given by Haefeli and Perla the primary fracture is in tension at the crown of the slab or in shear at the slab bottom. According to the proposed models, the fractures on the flanks of the slab follow the primary fracture. I am concerned that the explanation of the sawtooth fracture pattern on the flanks of the slab may require consideration of the dynamics of running cracks which is a problem in unstable equilibrium and may not be strongly dependent on pre-fracture conditions.

T. E. LANG: In other areas of mechanics similar results to these have been obtained. It is shown that failure does include other parameters but the shape of the slope before fracture is important. The failure criteria of other investigators do not contradict the theory given here.

R. J. Evans: In your introduction I believe you stated that the wave displacement you are considering is probably induced by creep and glide. Your potential energy expression, however, includes only terms due to displacement normal to the slope. Might not inclusion of potential energy terms due to down-slope displacement affect your results?

LANG: The dominant strain energy is in bending, so other displacements can be neglected provided there is not an accumulative force-transfer effect along the slope. The regular pattern of the sawtooth indicates a non-accumulative mechanism. 